\title{
Social Sustainability of High-rise Buildings
}

\author{
Mohammadjavad Mahdavinejad ${ }^{1}$, Ali Sadraie ${ }^{2, *}$, Golrokh Sadraie $^{3}$ \\ ${ }^{\mathbf{1}}$ Assistant Professor, Faculty of Art and Architecture, Tarbiat Modares University, Tehran, Iran \\ ${ }^{2}$ Master of Architecture, Kish International Campus, Tehran University, Shiraz, Iran \\ ${ }^{3}$ Master of Architecture, Faculty of Art and Architecture, Shiraz University, Shiraz, Iran \\ *Corresponding author: ali.sadraie@gmail.com
}

Received August 07, 2014; Revised November 26; Accepted March 03, 2014

\begin{abstract}
Nowadays, high-rise buildings have been proposed as a dominant form in world's major cities which its rapid growth has caused social and cultural concerns of the residents of these buildings. Social capital is remembered as a basis for economic development of any society. Its importance can be seen in the economic development of developing countries. Social capital is the invisible wealth of a country that encompasses institutions, relationships and norms that shape social interactions. In this paper, we try to achieve points in the social issues of these buildings with architectural principles and standards. For this purpose, we has been examined the relationship between the three elements of social capital, networks, confidence, partnership and architectural elements such as natural light, security, natural ventilation, visibility, space variability, physical identity, exterior solemnity, quality of entrance and lobby, quantity and quality of roadway and pedestrian access, beautiful landscaping and transfer of the annoying sounds in a number of samples. In this study we have chosen 9 high-rise building in Shiraz (one of the major cities of Iran) and to evaluate them in terms of social capital and architecture in two ways of observation and navigation (questioning residents by questionnaire and sampling) which finally substantially points were achieved in the design of high-rise buildings.
\end{abstract}

Keywords: social sustainability, social capital, high-rise buildings

Cite This Article: Mohammadjavad Mahdavinejad, Ali Sadraie, and Golrokh Sadraie, "Social Sustainability of High-rise Buildings." American Journal of Civil Engineering and Architecture, vol. 2, no. 1 (2014): 34-41. doi: 10.12691/ajcea-2-1-4.

\section{Introduction}

The advent of high-rise buildings in the twentieth century cities sky line is a result of increases in the price of land and technical innovations, but human invention as much as meeting the needs, is to achieve what they wish. Humans have always wanted to get into heaven and erected symbolic monument. Economic necessity of highrise buildings due to the increases in the land's price are just one factor to accelerate the creation of a building that is throughout the history terms like plying the ladder and reach the peak indicates the relative heights of honor and power.

Multitude of tall office buildings in the city center, not just a response to economic imperatives that also embodies the power of large corporations as well. This expression can be seen in the name that Frank Vinfild and Woolworth have denominated for Woolworth building: Cathedral of Commerce [6].

Although the high-rise building is result of complex process that it's elements have interplay and number of factors, including the characteristics of the cultural, social and economic influence in it but the principles and standards of architecture and urbanism could be established appropriate and desirable Usage of high-rise buildings. In general, according to particular circumstances of the present century appropriate and of course contingent usage of high-rise buildings could be considered as realistic and desirable solution to accommodate people and provide other needs related economic and social activities in large cities [2].

\subsection{Criticism of High-rise Buildings in Developed Countries (Pruitt-Igoe)}

In 1954 in the St. Louis (USA) a high-rise residential complex called Pruitt- Igoe was designed and built by Japanese architect, Minoru Yamasaki. Groups of Black and white and full children families settled in it, progressively many problems revealed in it as far as the number of units abandoned and finally 17 years after construction in 1972, it was decided to blast and demolish it. Many experts have explored the cause of this phenomenon that the result of one of these is: In general, high-rise residential buildings are appropriate for single people and young couples and small families and for families with many children, only under certain circumstances, such as a play space and lawn (green areas) on the roof in sufficient quantities, especially for children, is acceptable [1].

The other side, children whose living in high-rise buildings play outside the house less while the need for fresh air and social life for children means the need for playing outside of house. However, children's play area 
should be in adjacent or bilateral direct access for child to home or mother to child, so that Children easily have access to home security, while in the upper floors of tall buildings there are no close relationship between child and home. If the building's stories have communal terrace, these terraces and patio could have the function of playground for children. the other range of people who have a special status in high-rise buildings are elderly people, Due to their greater presence in the home than the community, they are in need of welfare and socialize, therefore the high-rise buildings which have appropriate communal spaces and sufficient green spaces are ideal for them, even the maintenance and care of green spaces in floors and roofs could be laid to them [4].

\subsection{High-rise Buildings in Developing Countries (Iran)}

Undoubtedly, the peak years of high-rise buildings in Iran is in the seventies and nineties which have special characteristics and factors unfortunately, in most cases, regardless of environmental conditions and other issues of social, cultural, economic etc. and have been implemented just like high-rise buildings in western countries in previous decades [2].

Theoretical framework of the paper focuses on highrise buildings in developing countries especially Iran. Community-oriented design for sustainability in architecture and planning [13] and focus on social issues [14] is to guarantee comprehensive approach toward environmental sustainability [15] and meet eco-friendly design and planning [16]. Developing countries such as Iran are in need of this comprehensive approach more than other parts of the world. [17,18] It seems that redevelopment plans in formal documents are more eco-friendly than it is in real perspectives [19].

\subsection{Problem Statement}

\subsubsection{Sustainability}

Sustainable design, a design that aims to answer the needs of today without damaging the resources of future generations. In the sustainable design, social and economic sustainability are important as energy consumption and environmental impact of buildings and urban areas [8].

\subsubsection{Social Capital}

Theories of social capital, relationships and social interactions are considered the most significant component of social capital, despite the differences in structure and content, what somehow collect these different approaches entitled to social theory, is the Issue of social interactions and relationships within the host network [7].

Table 1. Definitions of social capital

Table 1. Definitions of social capital
\begin{tabular}{|l|l|}
\hline James Coleman & $\begin{array}{l}\text { Aspect of social structure that facilitates } \\
\text { interactions of individuals within a social } \\
\text { structure [3]. }\end{array}$ \\
\hline Francis Fukuyama & $\begin{array}{l}\text { Set of social norms in the system that promote } \\
\text { cooperation members in that Society [5]. }\end{array}$ \\
\hline Spellerberg & $\begin{array}{l}\text { Social networks that increase the efficiency of } \\
\text { the functioning of society [11]. }\end{array}$ \\
\hline
\end{tabular}

In Table 1 a summary of conceptual scheme of social capital is represented.

Social capital consists of three elements of partnership, networks and confidence, which are as follows:

\subsubsection{Participation}

Includes the interaction of the individual and community groups to engage in self-determination and influence decision-making processes concerning public affairs [9].

\subsubsection{Network}

Social networks influence on the size and nature of social capital, the network of friends, family and neighbors up to participate in wider networks such as associations and voluntary groups are involved.

\subsubsection{Confidence}

Confidence is a sense each person has about that imperative which is approved [12].

This paper examines the three elements of social capital in the sample of and evaluate it in terms of architecture, in two methods of observation and examination of inhabitants (survey, questionnaire and sampling), at the end explains the relationship between the elements of social capital with desired architectural elements.

\subsection{Research Background}

In the field of internal research background, the following can be noted:

1- Bavan Puri, AR. The role of social capital in the a community sustainable development (Alley of Sajjadiye Mashhad), Master's thesis, Faculty of Sciences, Ferdowsi University of Mashhad, Supervisor BaratAli Khakpour, 1388. This paper, is based on look at asset-based, solution oriented, rather than need-based, merit-based and oriented as it is. Synergistic of social capital and community development, are based on component of this research and in Sajjadiye Alley in two cupped Martyr Ahmadi and Fakhar is measured in Mashhad council Zone 5. The method is a combination of documentary studies and surveys that have been used according to need. The method used is descriptive correlation analysis and questionnaire technique were tools to achieving the goals of research survey. However research shows after testing the research hypotheses based on findings obtained from the survey for the study area and according to observations researchers, it can be noted that the strengthening of social capital to achieve sustained neighborhood. However, a significant or nearly significant of some of the components of social capital can be stated that the concept of capital provided irrefutable objective of achieving sustainability, means achievement of sustainable development in the neighborhood,

Regardless of the humanistic and institutional potentials and benefiting from all individual and collective capacity is non-running affair [7].

In the field of foreign background can also be referred to:

1- Kontts: In his dissertation focuses on the relationship of corporate community, this study examines social capital and investment in neighborhoods within the city of Atlanta neighbors has been through a survey method. The 
researchers compared the levels of corporate enterprise of the local community with neighborhoods that are lacking in the company in the 1990s. Consistent with the empirical evidence shown in this study, neighborhoods with these companies than neighborhoods without these companies had comparable levels of investment [7].

2- Onyx \& Bullen (1997): To measure social capital in five locations in New South Wales, by employing a 68question survey began. They identified eight extensive elements in associated with social capital, That is: Participation in the local community, neighborhood connections, connections of family and friends, business connections, vitality in social context, sense of confidence and safety, tolerance of diversity and value of life. Onyx $\&$ Bullen found that in Comparison with three central region which surveyed, in all rural towns, considerably had higher levels of social capital But against the tolerance of diversity were not ranked enough high [10].

\section{Method}

\subsection{Examination: Samples (9 Buildings)}

In the survey method, the questionnaire has been prepared and in this questionnaire initially questions related to the three elements of social capital and then questions were outlined about those building's architecture which could not be respond by observation. (Due to not living in buildings).

\subsection{Questionnaire}

Table 2. Questionnaire

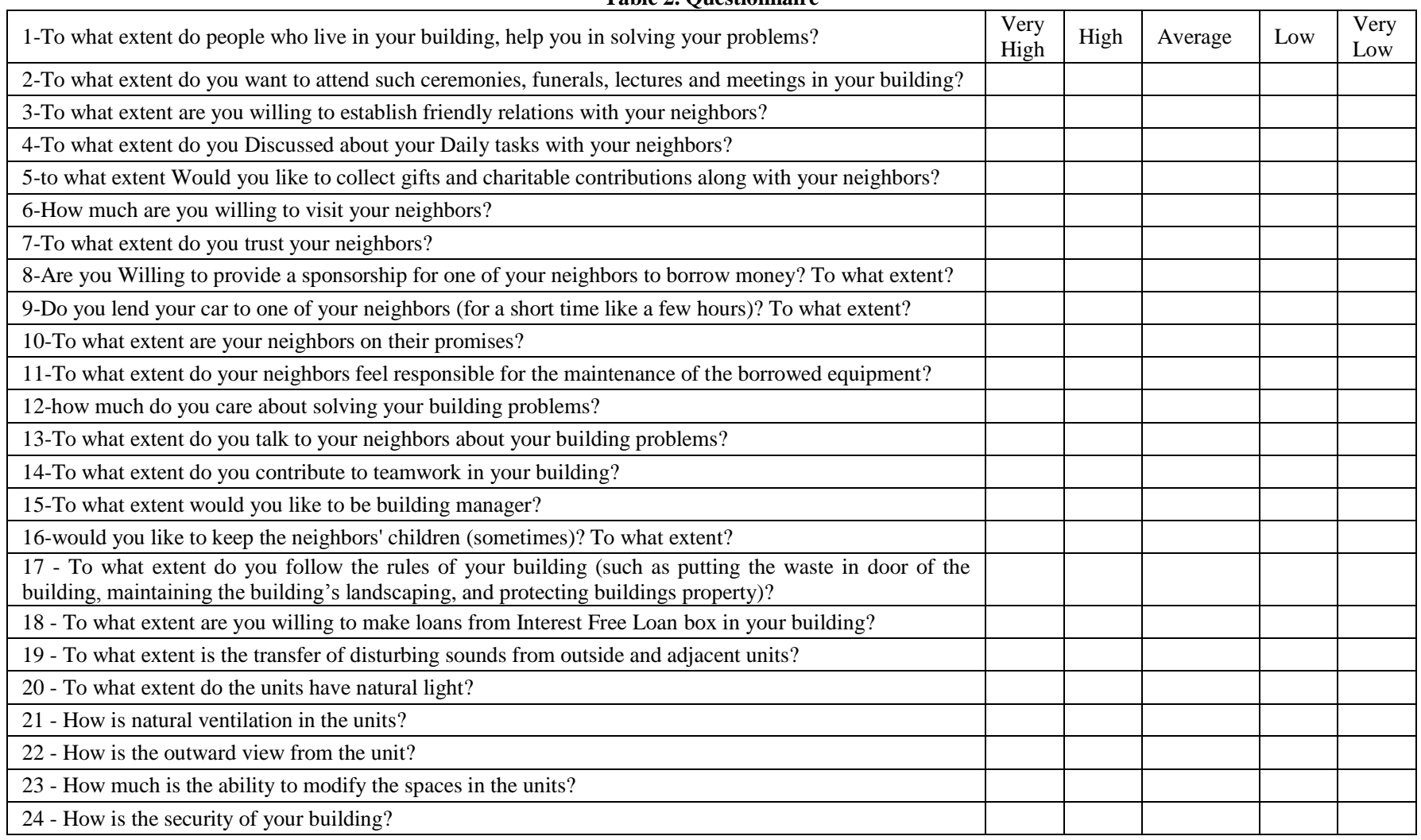

Table 3. Samples Evaluation (9 high-rise buildings)

\begin{tabular}{|c|c|c|c|c|c|c|c|c|}
\hline $\begin{array}{l}\text { Buildings } \\
\text { (Number of } \\
\text { completed } \\
\text { questionnaires) }\end{array}$ & $\begin{array}{l}\text { Physical } \\
\text { identity and } \\
\text { Exterior Awe } \\
(1-5)\end{array}$ & $\begin{array}{l}\text { Entry Quality, } \\
\text { Entrance and } \\
\text { lobby } \\
(1-5)\end{array}$ & $\begin{array}{l}\text { Common } \\
\text { areas: } \\
1 \text { - auditorium } \\
2 \text { - the pool } \\
4 \text { - gym }\end{array}$ & $\begin{array}{l}\text { Beautiful } \\
\text { landscaping } \\
(1-5)\end{array}$ & $\begin{array}{l}\text { Playground } \\
\text { for children }\end{array}$ & $\begin{array}{l}\text { Building } \\
\text { Services: } \\
1 \text { - Security } \\
2 \text { - Taxi }\end{array}$ & $\begin{array}{l}\text { Communal } \\
\text { space: } \\
1 \text { - Parking } \\
2 \text { - Storage } \\
3 \text { - Mechanical }\end{array}$ & $\begin{array}{l}\text { Quantity and } \\
\text { quality of the } \\
\text { cavalry and } \\
\text { infantry } \\
\text { Access } \\
(1-5)\end{array}$ \\
\hline BTM (12) & 4 & 5 & 1 & 5 & 1 & $0-1$ & $0-2-1$ & 2 \\
\hline Derak (8) & 3 & 4 & 1 & 2 & 1 & $0-1$ & $0-2-1$ & 3 \\
\hline Fardad (10) & 4 & 3 & 1 & 3 & 1 & $0-1$ & $0-2-1$ & 3 \\
\hline Moalemin (2) & 4 & 3 & 1 & 2 & - & $0-1$ & $1-2-3$ & 5 \\
\hline Pasargad (11) & 4 & 3 & 1 & 3 & - & $0-1$ & $1-2-3$ & 5 \\
\hline Bagro (6) & 4 & 2 & 1 & 4 & 1 & $0-1$ & $0-2-1$ & 4 \\
\hline Tandis (6) & 4 & 4 & - & 3 & 1 & $0-1$ & $1-2-3$ & 2 \\
\hline $\begin{array}{l}\text { Shaghayegh } \\
\text { (11) }\end{array}$ & 3 & 2 & 1 & 2 & 1 & $0-1$ & $0-2-1$ & 2 \\
\hline Shahed (11) & 5 & 5 & $1-2-3$ & 5 & 1 & $0-1$ & $0-2-1$ & 5 \\
\hline
\end{tabular}

\subsection{Samples Introduction}

11 case studies in Shiraz (one of the major cities in Iran) were selected (within an upper-middle class) and 15 questionnaires were distributed in each of these Samples and 9 building were cooperated. Buildings were evaluated in 8 cases of the architectural elements (through observation and field studies) in Table 3 . 


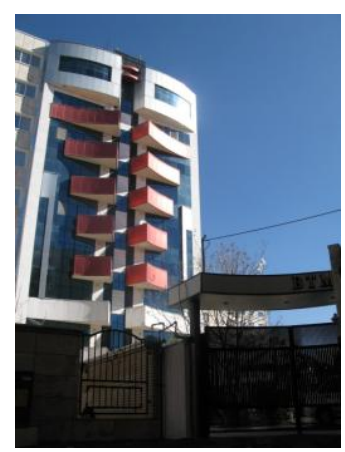

Figure 1. BTM

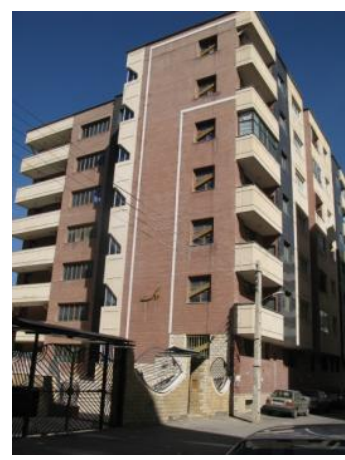

Figure 2. Derak

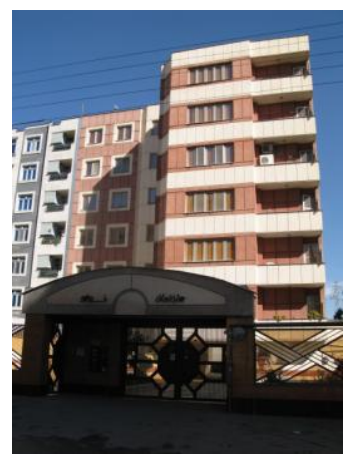

Figure 3. Fardad

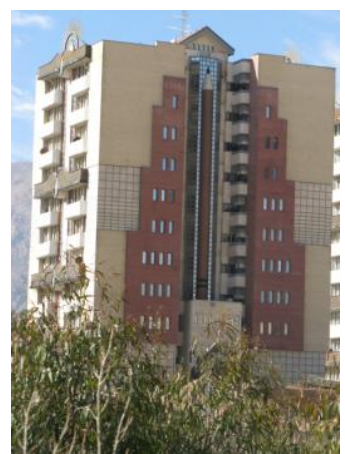

Figure 4. Moalemin

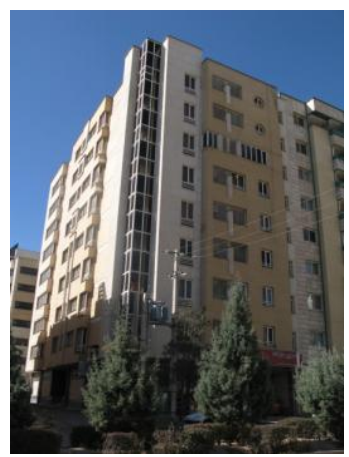

Figure 5. Pasargad

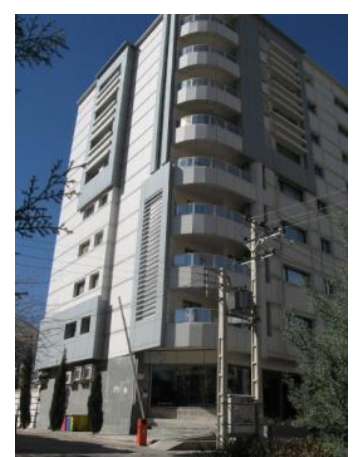

Figure 6. Bagro

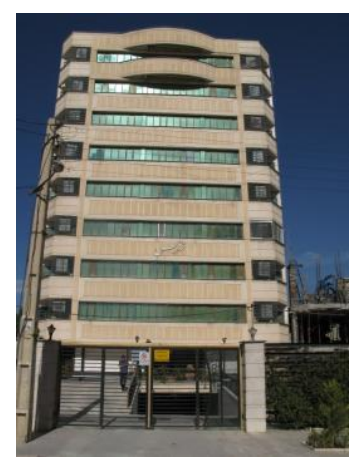

Figure 7. Tandis

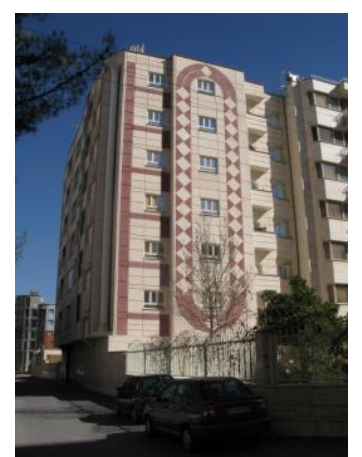

Figure 8. Shaghayegh

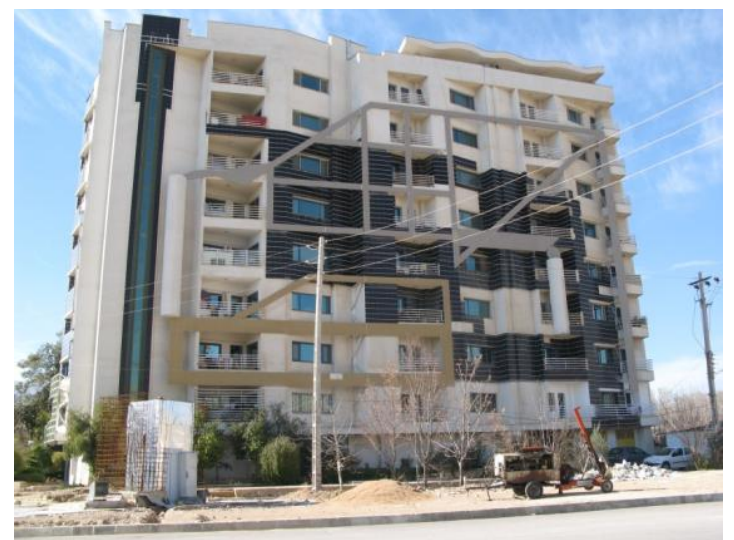

Figure 9. Shahed

\section{Results}

\subsection{Exam A}

Each building's questionnaire was evaluated and then the average and statistics of questions recorded by Microsoft Excel software and the overall average of all the buildings through SPSS software and the results of statistical operations of CORRELATION / PARTIAL is obtained as follows: 
Table 4. Output data from the SPSS software (by 28 items) correlations

\begin{tabular}{|c|c|c|c|c|c|c|c|c|c|c|c|c|c|c|c|}
\hline \multicolumn{3}{|c|}{ Control Variables } & Q1 & Q2 & Q3 & Q5 & Q6 & Q7 & Q8 & Q9 & Q10 & Q11 & Q12 & Q13 & Q14 \\
\hline \multirow{78}{*}{$\begin{array}{c}\text { Q15 \& } \\
\text { Q4 }\end{array}$} & Q1 & Correlation & 1.000 & .733 & .611 & .641 & .541 & .967 & .181 & -.408 & .819 & .706 & .579 & .565 & .659 \\
\hline & & Significance (2-tailed) & & .061 & .145 & .121 & .210 & .000 & .697 & .363 & .024 & .076 & .173 & .186 & .108 \\
\hline & & $\mathrm{df}$ & 0 & 5 & 5 & 5 & 5 & 5 & 5 & 5 & 5 & 5 & 5 & 5 & 5 \\
\hline & Q2 & Correlation & .733 & 1.000 & .609 & .547 & .704 & .790 & .223 & .043 & .785 & .799 & .891 & .786 & .827 \\
\hline & & Significance (2-tailed) & .061 & & .146 & .204 & .077 & .034 & .630 & .927 & .037 & .031 & .007 & .036 & .022 \\
\hline & & $\mathrm{df}$ & 5 & 0 & 5 & 5 & 5 & 5 & 5 & 5 & 5 & 5 & 5 & 5 & 5 \\
\hline & Q3 & Correlation & .611 & .609 & 1.000 & .556 & .314 & .509 & -.346 & -.124 & .822 & .629 & .590 & .080 & .213 \\
\hline & & Significance (2-tailed) & .145 & .146 & & .195 & .493 & .243 & .447 & .791 & .023 & .131 & .163 & .865 & .646 \\
\hline & & df & 5 & 5 & 0 & 5 & 5 & 5 & 5 & 5 & 5 & 5 & 5 & 5 & 5 \\
\hline & Q5 & Correlation & .641 & .547 & .556 & 1.000 & .388 & .551 & .387 & .248 & .851 & .374 & .321 & .131 & .482 \\
\hline & & Significance (2-tailed) & .121 & .204 & .195 & & .389 & .199 & .391 & .591 & .015 & .409 & .482 & .780 & .274 \\
\hline & & df & 5 & 5 & 5 & 0 & 5 & 5 & 5 & 5 & 5 & 5 & 5 & 5 & 5 \\
\hline & Q6 & Correlation & .541 & .704 & .314 & .388 & 1.000 & .594 & .403 & .140 & .415 & .338 & .751 & .556 & .848 \\
\hline & & Significance (2-tailed) & .210 & .077 & .493 & .389 & & .160 & .371 & .765 & .355 & .458 & .052 & .195 & .016 \\
\hline & & $\mathrm{df}$ & 5 & 5 & 5 & 5 & 0 & 5 & 5 & 5 & 5 & 5 & 5 & 5 & 5 \\
\hline & Q7 & Correlation & .967 & .790 & .509 & .551 & .594 & 1.000 & .170 & -.320 & .732 & .666 & .593 & .686 & .712 \\
\hline & & Significance (2-tailed) & .000 & .034 & .243 & .199 & .160 & & .715 & .484 & .061 & .103 & .160 & .089 & .073 \\
\hline & & $\mathrm{df}$ & 5 & 5 & 5 & 5 & 5 & 0 & 5 & 5 & 5 & 5 & 5 & 5 & 5 \\
\hline & Q8 & Correlation & .181 & 223 & -.346 & .387 & .403 & .170 & 1.000 & .124 & .161 & .152 & .227 & .431 & .669 \\
\hline & & Significance (2-tailed) & .697 & .630 & .447 & .391 & .371 & .715 & & .791 & .731 & .744 & .624 & .334 & .101 \\
\hline & & df & 5 & 5 & 5 & 5 & 5 & 5 & 0 & 5 & 5 & 5 & 5 & 5 & 5 \\
\hline & Q9 & Correlation & -.408 & .043 & -.124 & .248 & .140 & -.320 & .124 & 1.000 & -.080 & -.433 & -.106 & -.206 & -.003 \\
\hline & & Significance (2-tailed) & .363 & .927 & .791 & .591 & .765 & .484 & .791 & & .864 & .331 & .822 & .658 & .995 \\
\hline & & df & 5 & 5 & 5 & 5 & 5 & 5 & 5 & 0 & 5 & 5 & 5 & 5 & 5 \\
\hline & Q10 & Correlation & .819 & .785 & .822 & .851 & .415 & .732 & .161 & -.080 & 1.000 & .770 & .639 & .383 & .557 \\
\hline & & Significance (2-tailed) & .024 & .037 & .023 & .015 & .355 & .061 & .731 & .864 & & .043 & .123 & .396 & .194 \\
\hline & & df & 5 & 5 & 5 & 5 & 5 & 5 & 5 & 5 & 0 & 5 & 5 & 5 & 5 \\
\hline & Q11 & Correlation & .706 & .799 & .629 & .374 & .338 & .666 & .152 & -.433 & .770 & 1.000 & .817 & .709 & .616 \\
\hline & & Significance (2-tailed) & .076 & .031 & .131 & .409 & .458 & .103 & .744 & .331 & .043 & & .025 & .075 & .141 \\
\hline & & df & 5 & 5 & 5 & 5 & 5 & 5 & 5 & 5 & 5 & 0 & 5 & 5 & 5 \\
\hline & Q12 & Correlation & .579 & .891 & .590 & .321 & .751 & .593 & .227 & -.106 & .639 & .817 & 1.000 & .729 & .802 \\
\hline & & Significance (2-tailed) & .173 & .007 & .163 & .482 & .052 & .160 & .624 & .822 & .123 & .025 & & .063 & .030 \\
\hline & & $\mathrm{df}$ & 5 & 5 & 5 & 5 & 5 & 5 & 5 & 5 & 5 & 5 & 0 & 5 & 5 \\
\hline & Q13 & Correlation & .565 & .786 & .080 & .131 & .556 & .686 & .431 & -.206 & .383 & .709 & .729 & 1.000 & .839 \\
\hline & & Significance (2-tailed) & .186 & .036 & .865 & .780 & .195 & .089 & .334 & .658 & .396 & .075 & .063 & & .018 \\
\hline & & df & 5 & 5 & 5 & 5 & 5 & 5 & 5 & 5 & 5 & 5 & 5 & 0 & 5 \\
\hline & Q14 & Correlation & .659 & .827 & .213 & .482 & .848 & .712 & .669 & -.003 & .557 & .616 & .802 & .839 & 1.000 \\
\hline & & Significance (2-tailed) & .108 & .022 & .646 & .274 & .016 & .073 & .101 & .995 & .194 & .141 & .030 & .018 & \\
\hline & & df & 5 & 5 & 5 & 5 & 5 & 5 & 5 & 5 & 5 & 5 & 5 & 5 & 0 \\
\hline & Q16 & Correlation & .681 & .859 & .580 & .425 & .289 & .694 & .128 & -.241 & .777 & .950 & .754 & .758 & .607 \\
\hline & & Significance (2-tailed) & .092 & .013 & .173 & .342 & .529 & .084 & .785 & .602 & .040 & .001 & .050 & .048 & .148 \\
\hline & & $\mathrm{df}$ & 5 & 5 & 5 & 5 & 5 & 5 & 5 & 5 & 5 & 5 & 5 & 5 & 5 \\
\hline & Q17 & Correlation & .465 & .753 & .760 & .770 & .567 & .409 & .179 & .370 & .825 & .547 & .704 & .245 & .543 \\
\hline & & Significance (2-tailed) & .293 & .051 & .047 & .043 & .185 & .362 & .702 & .414 & .022 & .204 & .077 & .596 & .208 \\
\hline & & $\mathrm{df}$ & 5 & 5 & 5 & 5 & 5 & 5 & 5 & 5 & 5 & 5 & 5 & 5 & 5 \\
\hline & Q18 & Correlation & -.125 & .229 & .343 & .413 & .494 & -.150 & .083 & .738 & .220 & -.159 & .285 & -.240 & .180 \\
\hline & & Significance (2-tailed) & .789 & .622 & .451 & .357 & .260 & .748 & .860 & .058 & .635 & .734 & .535 & .605 & .699 \\
\hline & & df & 5 & 5 & 5 & 5 & 5 & 5 & 5 & 5 & 5 & 5 & 5 & 5 & 5 \\
\hline & Q19 & Correlation & .075 & .687 & .386 & .261 & .231 & .165 & .003 & .480 & .438 & .497 & .591 & .463 & .366 \\
\hline & & Significance (2-tailed) & .872 & .088 & .392 & .572 & .618 & .724 & .995 & .276 & .326 & .256 & .162 & .295 & .419 \\
\hline & & df & 5 & 5 & 5 & 5 & 5 & 5 & 5 & 5 & 5 & 5 & 5 & 5 & 5 \\
\hline & Q20 & Correlation & .763 & .804 & .769 & .488 & .613 & .684 & .162 & -.358 & .814 & .892 & .900 & .560 & .686 \\
\hline & & Significance (2-tailed) & .046 & .029 & .043 & .267 & .143 & .090 & .729 & .430 & .026 & .007 & .006 & .191 & .089 \\
\hline & & $\mathrm{df}$ & 5 & 5 & 5 & 5 & 5 & 5 & 5 & 5 & 5 & 5 & 5 & 5 & 5 \\
\hline & Q21 & Correlation & .488 & .831 & .556 & .601 & .328 & .477 & .306 & .133 & .798 & .819 & .736 & .605 & .618 \\
\hline & & Significance (2-tailed) & .267 & .020 & .195 & .154 & .473 & .279 & .504 & .777 & .031 & .024 & .059 & .150 & .139 \\
\hline & & $\mathrm{df}$ & 5 & 5 & 5 & 5 & 5 & 5 & 5 & 5 & 5 & 5 & 5 & 5 & 5 \\
\hline & Q22 & Correlation & -.030 & -.150 & .272 & .453 & -.376 & -.247 & .202 & -.039 & .377 & .193 & -.095 & -.379 & -.189 \\
\hline & & Significance (2-tailed) & .950 & .748 & .555 & .308 & .405 & .594 & .665 & .934 & .404 & .678 & .839 & .401 & .685 \\
\hline & & df & 5 & 5 & 5 & 5 & 5 & 5 & 5 & 5 & 5 & 5 & 5 & 5 & 5 \\
\hline & Q23 & Correlation & .137 & .095 & .550 & .667 & -.302 & .017 & -.198 & .320 & .568 & .111 & -.124 & -.397 & -.251 \\
\hline & & Significance (2-tailed) & .770 & .840 & .201 & .101 & .510 & .971 & .671 & .484 & .183 & .813 & .791 & .378 & .588 \\
\hline & & df & 5 & 5 & 5 & 5 & 5 & 5 & 5 & 5 & 5 & 5 & 5 & 5 & 5 \\
\hline & Q24 & Correlation & .641 & .704 & .504 & .749 & .659 & .545 & .628 & -.022 & .790 & .674 & .742 & .475 & .802 \\
\hline & & Significance (2-tailed) & .121 & .077 & .249 & .053 & .107 & .205 & .131 & .962 & .034 & .097 & .056 & .281 & .030 \\
\hline & & df & 5 & 5 & 5 & 5 & 5 & 5 & 5 & 5 & 5 & 5 & 5 & 5 & 5 \\
\hline & Q25 & Correlation & -.011 & .190 & .532 & -.387 & -.078 & -.024 & -.709 & -.334 & .083 & .408 & .393 & .045 & -.204 \\
\hline & & Significance (2-tailed) & .981 & .683 & .219 & .392 & .869 & .959 & .074 & .464 & .859 & .364 & .383 & .924 & .661 \\
\hline & & $\mathrm{df}$ & 5 & 5 & 5 & 5 & 5 & 5 & 5 & 5 & 5 & 5 & 5 & 5 & 5 \\
\hline & Q26 & Correlation & .004 & .080 & .535 & .000 & -.015 & .042 & -.847 & .253 & .102 & -.149 & -.027 & -.350 & -.379 \\
\hline & & Significance (2-tailed) & .993 & .865 & .216 & .999 & .975 & .930 & .016 & .584 & .828 & .750 & .955 & .441 & .402 \\
\hline & & $\mathrm{df}$ & 5 & 5 & 5 & 5 & 5 & 5 & 5 & 5 & 5 & 5 & 5 & 5 & 5 \\
\hline & Q27 & Correlation & -.174 & .072 & .280 & -.534 & -.262 & -.091 & -.834 & -.146 & -.141 & .162 & .122 & .026 & -.369 \\
\hline & & Significance (2-tailed) & .710 & .877 & .543 & .217 & .570 & .847 & .020 & .754 & .762 & .729 & .794 & .955 & .415 \\
\hline & & $\mathrm{df}$ & 5 & 5 & 5 & 5 & 5 & 5 & 5 & 5 & 5 & 5 & 5 & 5 & 5 \\
\hline & Q28 & Correlation & -.113 & -.105 & .310 & -.322 & -.390 & -.249 & -.330 & -.570 & .058 & .437 & .187 & -.094 & -.287 \\
\hline & & Significance (2-tailed) & .809 & .824 & .499 & .481 & .387 & .591 & .470 & .181 & .902 & .327 & .688 & .842 & .532 \\
\hline & & $\mathrm{df}$ & 5 & 5 & 5 & 5 & 5 & 5 & 5 & 5 & 5 & 5 & 5 & 5 & 5 \\
\hline
\end{tabular}




\begin{tabular}{|c|c|c|c|c|c|c|c|c|c|c|c|c|c|c|c|}
\hline \multirow{3}{*}{$\begin{array}{c}\text { Q15 \& } \\
\text { Q4 }\end{array}$} & \multicolumn{2}{|c|}{ Control Variables } & Q16 & Q17 & Q18 & Q19 & Q20 & Q21 & Q22 & Q23 & Q24 & Q25 & Q26 & Q27 & $\mathrm{Q} 28$ \\
\hline & Q1 & Correlation & .681 & .465 & -.125 & .075 & .763 & .488 & -.030 & .137 & .641 & -.011 & .004 & -.174 & -.113 \\
\hline & & Significance (2-tailed) & .092 & .293 & .789 & .872 & .046 & .267 & .950 & .770 & .121 & .981 & .993 & .710 & .809 \\
\hline & & $\mathrm{df}$ & 5 & 5 & 5 & 5 & 5 & 5 & 5 & 5 & 5 & 5 & 5 & 5 & 5 \\
\hline & Q2 & Correlation & .859 & .753 & .229 & .687 & .804 & .831 & -.150 & .095 & .704 & .190 & .080 & .072 & -.105 \\
\hline & & Significance (2-tailed) & .013 & .051 & .622 & .088 & .029 & .020 & .748 & .840 & .077 & .683 & .865 & .877 & .824 \\
\hline & & df & 5 & 5 & 5 & 5 & 5 & 5 & 5 & 5 & 5 & 5 & 5 & 5 & 5 \\
\hline & Q3 & Correlation & .580 & .760 & .343 & .386 & .769 & .556 & .272 & .550 & .504 & .532 & .535 & .280 & .310 \\
\hline & & Significance (2-tailed) & .173 & .047 & .451 & .392 & .043 & .195 & .555 & .201 & .249 & .219 & .216 & .543 & .499 \\
\hline & & df & 5 & 5 & 5 & 5 & 5 & 5 & 5 & 5 & 5 & 5 & 5 & 5 & 5 \\
\hline & Q5 & Correlation & .425 & .770 & .413 & .261 & .488 & .601 & .453 & .667 & .749 & -.387 & .000 & -.534 & -.322 \\
\hline & & Significance (2-tailed) & .342 & .043 & .357 & .572 & .267 & .154 & .308 & .101 & .053 & .392 & .999 & .217 & .481 \\
\hline & & $\mathrm{df}$ & 5 & 5 & 5 & 5 & 5 & 5 & 5 & 5 & 5 & 5 & 5 & 5 & 5 \\
\hline & Q6 & Correlation & 289 & .567 & .494 & 231 & .613 & .328 & -.376 & -.302 & 659 & -.078 & -.015 & -.262 & -.390 \\
\hline & & Significance (2-tailed) & .529 & .185 & .260 & .618 & .143 & .473 & .405 & .510 & .107 & .869 & .975 & .570 & .387 \\
\hline & & df & 5 & 5 & 5 & 5 & 5 & 5 & 5 & 5 & 5 & 5 & 5 & 5 & 5 \\
\hline & Q7 & Correlation & .694 & .409 & -.150 & 165 & .684 & .477 & -.247 & .017 & .545 & -.024 & .042 & -.091 & -.249 \\
\hline & & Significance (2-tailed) & .084 & .362 & .748 & .724 & .090 & .279 & .594 & .971 & .205 & .959 & .930 & .847 & .591 \\
\hline & & df & 5 & 5 & 5 & 5 & 5 & 5 & 5 & 5 & 5 & 5 & 5 & 5 & 5 \\
\hline & Q8 & Correlation & .128 & .179 & .083 & .003 & .162 & .306 & .202 & -.198 & .628 & -.709 & -.847 & -.834 & -.330 \\
\hline & & Significance (2-tailed) & .785 & .702 & .860 & .995 & .729 & .504 & .665 & .671 & .131 & .074 & .016 & .020 & .470 \\
\hline & & df & 5 & 5 & 5 & 5 & 5 & 5 & 5 & 5 & 5 & 5 & 5 & 5 & 5 \\
\hline & Q9 & Correlation & -.241 & 370 & .738 & .480 & -.358 & .133 & -.039 & .320 & -.022 & -.334 & .253 & -.146 & -.570 \\
\hline & & Significance (2-tailed) & .602 & .414 & .058 & .276 & .430 & .777 & .934 & .484 & .962 & .464 & .584 & .754 & .181 \\
\hline & & df & 5 & 5 & 5 & 5 & 5 & 5 & 5 & 5 & 5 & 5 & 5 & 5 & 5 \\
\hline & Q10 & Correlation & .777 & .825 & .220 & .438 & .814 & .798 & .377 & .568 & .790 & .083 & .102 & -.141 & .058 \\
\hline & & Significance (2-tailed) & .040 & .022 & .635 & .326 & .026 & .031 & .404 & .183 & .034 & .859 & .828 & .762 & .902 \\
\hline & & df & 5 & 5 & 5 & 5 & 5 & 5 & 5 & 5 & 5 & 5 & 5 & 5 & 5 \\
\hline & Q11 & Correlation & .950 & .547 & -.159 & .497 & .892 & .819 & .193 & .111 & .674 & .408 & -.149 & 162 & 437 \\
\hline & & Significance (2-tailed) & .001 & .204 & .734 & .256 & .007 & .024 & .678 & .813 & .097 & .364 & .750 & .729 & 327 \\
\hline & & df & 5 & 5 & 5 & 5 & 5 & 5 & 5 & 5 & 5 & 5 & 5 & 5 & 5 \\
\hline & Q12 & Correlation & .754 & .704 & .285 & .591 & .900 & .736 & -.095 & -.124 & .742 & .393 & -.027 & .122 & 187 \\
\hline & & Significance (2-tailed) & .050 & .077 & .535 & .162 & .006 & .059 & .839 & .791 & .056 & .383 & .955 & .794 & 688 \\
\hline & & df & 5 & 5 & 5 & 5 & 5 & 5 & 5 & 5 & 5 & 5 & 5 & 5 & 5 \\
\hline & Q13 & Correlation & .758 & .245 & -.240 & .463 & .560 & .605 & -.379 & -.397 & .475 & .045 & -.350 & .026 & -.094 \\
\hline & & Significance (2-tailed) & .048 & .596 & .605 & .295 & .191 & .150 & .401 & .378 & .281 & .924 & .441 & .955 & .842 \\
\hline & & $\mathrm{df}$ & 5 & 5 & 5 & 5 & 5 & 5 & 5 & 5 & 5 & 5 & 5 & 5 & 5 \\
\hline & Q14 & Correlation & .607 & .543 & .180 & .366 & .686 & .618 & -.189 & -.251 & .802 & -.204 & -.379 & -.369 & -.287 \\
\hline & & Significance (2-tailed) & .148 & .208 & .699 & .419 & .089 & .139 & .685 & .588 & .030 & .661 & .402 & .415 & .532 \\
\hline & & df & 5 & 5 & 5 & 5 & 5 & 5 & 5 & 5 & 5 & 5 & 5 & 5 & 5 \\
\hline & Q16 & Correlation & 1.000 & .567 & -.151 & .659 & .768 & .891 & .106 & .209 & .586 & .325 & -.062 & .211 & 257 \\
\hline & & Significance (2-tailed) & & .184 & .746 & .107 & .044 & .007 & .822 & .653 & .167 & .477 & .895 & .650 & .578 \\
\hline & & $\mathrm{df}$ & 0 & 5 & 5 & 5 & 5 & 5 & 5 & 5 & 5 & 5 & 5 & 5 & 5 \\
\hline & Q17 & Correlation & .567 & 1.000 & .698 & .663 & .704 & .791 & .325 & .535 & .782 & .122 & .222 & -.111 & -.031 \\
\hline & & Significance (2-tailed) & .184 & & .081 & .104 & .077 & .034 & .476 & .216 & .038 & .794 & .633 & .813 & .948 \\
\hline & & $\mathrm{df}$ & 5 & 0 & 5 & 5 & 5 & 5 & 5 & 5 & 5 & 5 & 5 & 5 & 5 \\
\hline & Q18 & Correlation & -.151 & .698 & 1.000 & .377 & .159 & .213 & .127 & .317 & .371 & -.040 & .346 & -.176 & -.272 \\
\hline & & Significance (2-tailed) & .746 & .081 & & .404 & .733 & .647 & .785 & .489 & .413 & .933 & .447 & .706 & .555 \\
\hline & & $\mathrm{df}$ & 5 & 5 & 0 & 5 & 5 & 5 & 5 & 5 & 5 & 5 & 5 & 5 & 5 \\
\hline & Q19 & Correlation & .659 & .663 & .377 & 1.000 & .359 & .816 & .008 & .303 & .317 & .323 & .216 & .361 & .047 \\
\hline & & Significance (2-tailed) & .107 & .104 & .404 & & .430 & .025 & .987 & .509 & 489 & .480 & .641 & .426 & 921 \\
\hline & & $\mathrm{df}$ & 5 & 5 & 5 & 0 & 5 & 5 & 5 & 5 & 5 & 5 & 5 & 5 & 5 \\
\hline & Q20 & Correlation & .768 & .704 & .159 & .359 & 1.000 & .698 & .172 & .089 & .815 & .384 & -.024 & .021 & .335 \\
\hline & & Significance (2-tailed) & .044 & .077 & .733 & .430 & & .081 & .713 & .850 & .025 & .395 & .959 & .964 & 463 \\
\hline & & $\mathrm{df}$ & 5 & 5 & 5 & 5 & 0 & 5 & 5 & 5 & 5 & 5 & 5 & 5 & 5 \\
\hline & Q21 & Correlation & .891 & .791 & .213 & .816 & .698 & 1.000 & .326 & .404 & .726 & .156 & -.107 & .009 & .158 \\
\hline & & Significance (2-tailed) & .007 & .034 & .647 & .025 & .081 & & .476 & .368 & .065 & .739 & .819 & .985 & 736 \\
\hline & & $\mathrm{df}$ & 5 & 5 & 5 & 5 & 5 & 0 & 5 & 5 & 5 & 5 & 5 & 5 & 5 \\
\hline & Q22 & Correlation & .106 & .325 & .127 & .008 & .172 & .326 & 1.000 & .679 & .391 & -.094 & -.270 & -.352 & .461 \\
\hline & & Significance (2-tailed) & .822 & .476 & .785 & .987 & .713 & .476 & & .093 & .386 & .842 & .558 & .439 & .298 \\
\hline & & $\mathrm{df}$ & 5 & 5 & 5 & 5 & 5 & 5 & 0 & 5 & 5 & 5 & 5 & 5 & 5 \\
\hline & Q23 & Correlation & .209 & .535 & .317 & .303 & .089 & .404 & .679 & 1.000 & .199 & -.032 & .364 & -.055 & .055 \\
\hline & & Significance (2-tailed) & .653 & .216 & .489 & .509 & .850 & .368 & .093 & & .668 & .945 & .422 & .908 & .907 \\
\hline & & df & 5 & 5 & 5 & 5 & 5 & 5 & 5 & 0 & 5 & 5 & 5 & 5 & 5 \\
\hline & Q24 & Correlation & .586 & .782 & .371 & .317 & .815 & .726 & .391 & .199 & 1.000 & -.150 & -.352 & -.503 & .015 \\
\hline & & Significance (2-tailed) & .167 & .038 & .413 & .489 & .025 & .065 & .386 & .668 & & .748 & .438 & .250 & .975 \\
\hline & & $\mathrm{df}$ & 5 & 5 & 5 & 5 & 5 & 5 & 5 & 5 & 0 & 5 & 5 & 5 & 5 \\
\hline & Q25 & Correlation & .325 & .122 & -.040 & .323 & .384 & .156 & -.094 & -.032 & -.150 & 1.000 & .517 & .875 & .726 \\
\hline & & Significance (2-tailed) & .477 & .794 & .933 & .480 & .395 & .739 & .842 & .945 & .748 & & .234 & .010 & .065 \\
\hline & & df & 5 & 5 & 5 & 5 & 5 & 5 & 5 & 5 & 5 & 0 & 5 & 5 & 5 \\
\hline & Q26 & Correlation & -.062 & .222 & .346 & .216 & -.024 & -.107 & -.270 & .364 & -.352 & .517 & 1.000 & .640 & -.052 \\
\hline & & Significance (2-tailed) & .895 & .633 & .447 & .641 & .959 & .819 & .558 & .422 & .438 & .234 & & .121 & .912 \\
\hline & & $\mathrm{df}$ & 5 & 5 & 5 & 5 & 5 & 5 & 5 & 5 & 5 & 5 & 0 & 5 & 5 \\
\hline & Q27 & Correlation & .211 & -.111 & -.176 & .361 & .021 & .009 & -.352 & -.055 & -.503 & .875 & .640 & 1.000 & .459 \\
\hline & & Significance (2-tailed) & .650 & .813 & .706 & .426 & .964 & .985 & .439 & .908 & .250 & .010 & .121 & & .300 \\
\hline & & df & 5 & 5 & 5 & 5 & 5 & 5 & 5 & 5 & 5 & 5 & 5 & 0 & 5 \\
\hline & Q28 & Correlation & .257 & -.031 & -.272 & .047 & .335 & .158 & .461 & .055 & .015 & .726 & -.052 & .459 & 1.000 \\
\hline & & Significance (2-tailed) & .578 & .948 & .555 & .921 & .463 & .736 & .298 & .907 & .975 & .065 & .912 & .300 & \\
\hline & & df & 5 & 5 & 5 & 5 & 5 & 5 & 5 & 5 & 5 & 5 & 5 & 5 & 0 \\
\hline
\end{tabular}




\subsection{Exam B}

In this exam questions of social field are summarized in three categories of network, confidence and partnership to achieve a more general conclusions.

Q’1=Q1-Q6 ‘Q’2=Q7-Q11 ‘Q’3=Q19-Q24

Table 5. Output data of the SPSS software ( 13 items) correlations

\begin{tabular}{|c|c|c|c|c|c|c|c|c|c|c|c|c|c|c|}
\hline \multicolumn{3}{|c|}{ Control Variables } & $\mathrm{Q}^{\prime} 1$ & $\mathrm{Q}^{\prime} 2$ & $\mathrm{Q}^{\prime} 3$ & $\mathrm{Q}^{\prime} 5$ & $\mathrm{Q}^{\prime} 6$ & Q'7 & $\mathrm{Q}^{\prime} 8$ & $\mathrm{Q}^{\prime} 9$ & $\mathrm{Q}^{\prime} 10$ & $\mathrm{Q}^{\prime} 11$ & $\mathrm{Q}^{\prime} 12$ & $\mathrm{Q}^{\prime} 13$ \\
\hline \multirow[t]{36}{*}{$\mathrm{Q}^{\prime} 4$} & \multirow[t]{3}{*}{$\mathrm{Q}^{\prime} 1$} & Correlation & 1.000 & .949 & .685 & .788 & .577 & .453 & .219 & .520 & -.145 & .341 & .009 & .015 \\
\hline & & Significance (2-tailed) & & .000 & .061 & .020 & .135 & .259 & .603 & .186 & .733 & .408 & .983 & .973 \\
\hline & & $\mathrm{df}$ & 0 & 6 & 6 & 6 & 6 & 6 & 6 & 6 & 6 & 6 & 6 & 6 \\
\hline & \multirow[t]{3}{*}{$\mathrm{Q}^{\prime 2}$} & Correlation & .949 & 1.000 & .763 & .775 & .624 & .520 & .181 & .540 & -.225 & .085 & -.028 & .030 \\
\hline & & Significance (2-tailed) & .000 & & .028 & .024 & .098 & .186 & .668 & .167 & .592 & .842 & .947 & .943 \\
\hline & & df & 6 & 0 & 6 & 6 & 6 & 6 & 6 & 6 & 6 & 6 & 6 & 6 \\
\hline & \multirow[t]{3}{*}{ Q'3 } & Correlation & .685 & .763 & 1.000 & .666 & .225 & .314 & -.002 & .481 & -.116 & -.130 & .043 & -.186 \\
\hline & & Significance (2-tailed) & .061 & .028 & & .071 & .592 & .449 & .997 & .227 & .785 & .758 & .919 & .659 \\
\hline & & $\mathrm{df}$ & 6 & 6 & 0 & 6 & 6 & 6 & 6 & 6 & 6 & 6 & 6 & 6 \\
\hline & \multirow[t]{3}{*}{ Q'5 } & Correlation & .788 & .775 & .666 & 1.000 & .731 & .310 & -.016 & .732 & .255 & .115 & -.016 & .415 \\
\hline & & Significance (2-tailed) & .020 & .024 & .071 & & .039 & .455 & .969 & .039 & .541 & .787 & .971 & .307 \\
\hline & & $\mathrm{df}$ & 6 & 6 & 6 & 0 & 6 & 6 & 6 & 6 & 6 & 6 & 6 & 6 \\
\hline & \multirow[t]{3}{*}{ Q'6 } & Correlation & .577 & .624 & .225 & .731 & 1.000 & .320 & .102 & .815 & -.132 & -.166 & -.484 & .398 \\
\hline & & Significance (2-tailed) & .135 & .098 & .592 & .039 & & .440 & .811 & .014 & .755 & .694 & .224 & .329 \\
\hline & & $\mathrm{df}$ & 6 & 6 & 6 & 6 & 0 & 6 & 6 & 6 & 6 & 6 & 6 & 6 \\
\hline & \multirow[t]{3}{*}{ Q'7 } & Correlation & .453 & .520 & .314 & .310 & .320 & 1.000 & .670 & .231 & -.132 & -.013 & .034 & .319 \\
\hline & & Significance (2-tailed) & .259 & .186 & .449 & .455 & .440 & & .069 & .582 & .755 & .975 & .937 & .441 \\
\hline & & df & 6 & 6 & 6 & 6 & 6 & 0 & 6 & 6 & 6 & 6 & 6 & 6 \\
\hline & \multirow[t]{3}{*}{ Q'8 } & Correlation & .219 & .181 & -.002 & -.016 & .102 & .670 & 1.000 & .030 & -.162 & .281 & -.021 & -.038 \\
\hline & & Significance (2-tailed) & .603 & .668 & .997 & .969 & .811 & .069 & & .944 & .701 & .500 & .960 & .928 \\
\hline & & $\mathrm{df}$ & 6 & 6 & 6 & 6 & 6 & 6 & 0 & 6 & 6 & 6 & 6 & 6 \\
\hline & \multirow[t]{3}{*}{$\mathrm{Q}^{\prime} 9$} & Correlation & .520 & .540 & .481 & .732 & .815 & .231 & .030 & 1.000 & -.248 & -.333 & -.680 & .113 \\
\hline & & Significance (2-tailed) & .186 & .167 & .227 & .039 & .014 & .582 & .944 & & .554 & .421 & .063 & .790 \\
\hline & & df & 6 & 6 & 6 & 6 & 6 & 6 & 6 & 0 & 6 & 6 & 6 & 6 \\
\hline & \multirow[t]{3}{*}{$\mathrm{Q}^{\prime} 10$} & Correlation & -.145 & -.225 & -.116 & .255 & -.132 & -.132 & -.162 & -.248 & 1.000 & .418 & .697 & .699 \\
\hline & & Significance (2-tailed) & .733 & .592 & .785 & .541 & .755 & .755 & .701 & .554 & & .302 & .055 & .054 \\
\hline & & $\mathrm{df}$ & 6 & 6 & 6 & 6 & 6 & 6 & 6 & 6 & 0 & 6 & 6 & 6 \\
\hline & \multirow[t]{3}{*}{$\mathrm{Q}^{\prime} 11$} & Correlation & .341 & .085 & -.130 & .115 & -.166 & -.013 & .281 & -.333 & .418 & 1.000 & .561 & .080 \\
\hline & & Significance (2-tailed) & .408 & .842 & .758 & .787 & .694 & .975 & .500 & .421 & .302 & & .148 & .851 \\
\hline & & $\mathrm{df}$ & 6 & 6 & 6 & 6 & 6 & 6 & 6 & 6 & 6 & 0 & 6 & 6 \\
\hline & \multirow[t]{3}{*}{$\mathrm{Q}^{\prime} 12$} & Correlation & .009 & -.028 & .043 & -.016 & -.484 & .034 & -.021 & -.680 & .697 & .561 & 1.000 & .300 \\
\hline & & Significance (2-tailed) & .983 & .947 & .919 & .971 & .224 & .937 & .960 & .063 & .055 & .148 & & .470 \\
\hline & & df & 6 & 6 & 6 & 6 & 6 & 6 & 6 & 6 & 6 & 6 & 0 & 6 \\
\hline & \multirow[t]{3}{*}{$\mathrm{Q}^{\prime} 13$} & Correlation & .015 & .030 & -.186 & .415 & .398 & .319 & -.038 & .113 & .699 & .080 & .300 & 1.000 \\
\hline & & Significance (2-tailed) & .973 & .943 & .659 & .307 & .329 & .441 & .928 & .790 & .054 & .851 & .470 & \\
\hline & & $\mathrm{df}$ & 6 & 6 & 6 & 6 & 6 & 6 & 6 & 6 & 6 & 6 & 6 & 0 \\
\hline
\end{tabular}

Q24-Q27 -0.503: Buildings that are more secure, they

\section{Discussion}

\subsection{Exam A}

Q11-Q20 0.892: When the Units have more light, Residents have been more responsible than their neighbors.

Q12-Q20 0.9: When the Units have more light, Tend to solve problems is more common among the inhabitants of the building.

Q13-Q22 -0.379: In Buildings which units have wider outward view, Residents have been more inclined to talk to neighbors about construction problems.

Q22-Q27 -0.352: In Buildings which units have better outward view, frequently there is less green spaces.

Q16-Q21 0.891: In Buildings which units have better natural ventilation, Contribution to keep the neighbors' children are more.

Q19-Q21 0.816: In Buildings which units have better natural ventilation, Disturbing sounds of the outside and adjacent units are more.

Q13-Q26 -0.350: Buildings that have better quality of entry and lobbies, Neighbors speak less about the buildings problems with each other.

Q24-Q26 -0.352: Buildings that have better quality in entries and lobbies, building security is less effective. have less beautiful landscaping.

Q8-Q27 -0.834: In Buildings that have been more and beautiful green space, Inhabitants trust less on each other.

Q25-Q27 0.875: Buildings that have prettier and greener space, also had a better exterior solemnity and somatogenic identity.

Q8-Q28 -0.330: In Buildings with better access to Roadway and pedestrian way, Inhabitants trust less on each other.

Q5-Q28 -0.322: In Buildings with better access to Roadway and pedestrian way, Social network and helping neighbors are less effective in them.

Q8-Q25 -0.709: Buildings that have a better exterior solemnity and somatogenic identity, Inhabitants trust (sponsorship) is lower in them.

Q13-Q23 -0.397: In Buildings that have more variability, Inhabitants speak less with neighbors about buildings problems.

\subsection{Exam B}

In the Exam B Items of networks, confidence and partnership are identical, which have direct relation with natural light, security, natural ventilation, visibility and space variability and are inversely related to the physical identity, exterior solemnity, quality of entrance and lobby, 
quantity and quality of roadway and pedestrian access, beautiful landscaping and transfer of the annoying sounds.

\section{Conclusions}

According to the survey, the following results were achieved:

1-In buildings with better physical identity and solemnity exterior, green landscape and good quality in lobby and entrance, security is felt less, this indicates that pay attention to building's appearance is not merely enough and in building design, in addition to beauty, security must also be considered.

2-The inappropriate quantity and quality of the roadway and pedestrian access show that the building was away from the main street and social participation seen more in it, so in the layout of buildings, auxiliary road, is more appropriate.

3-In that building that each unit is flexible (the ability to customize the space to inhabitants), neighbors participation in the building problems are rarely seen, thus, designing each unit with detail and flexible manner, to increase social participation among inhabitants must be considered.

4-In the buildings that have better quality of entries and lobby space, neighbors participation in the building problems are rarely seen, so if the quality of the entrance and lobby area designed to be good and somehow flexible and allow inhabitants to provide the desired change, lead to further participation in the building affairs by its neighbors.

5-In the buildings that are wider outside view, beautiful landscape spaces are less observed, these two factors have acted individually, therefore the view to the beautiful landscape spaces in buildings should be provided so the visually feature would be more efficient to use.

\section{References}

[1] Aregger, H., \& Glaus, O., High-rise building and urban design, FA Praeger, 1967.
[2] Bemanian, MR, High-rise buildings and the city's. First edition, Nashr Shahr institute in Tehran, 2011.

[3] Coleman James S, Foundations of social theory, 1994.

[4] Conway,Donald J, Human response to tall building. Hutchinston and Ross Inc, 1977.

[5] Fukuyama, F, The end of order. London: Social Market Foundation, 1997.

[6] Kohn, E, The tall Building. In Proceedings of 4th World Congress on Tall Buildings: Tall Buildings, translated by Farzaneh Taheri, Quoted from the book: on the fundamental issues of Tall building, 2000, 17-36.

[7] Khavari, Zahra, The effect of social capital indicators on economic conditions of Neighborhood Case study: Tollab Neighborhood, Municipal management letter especial edition, 2011: 277-298.

[8] Lang, J. T, Creating architectural theory: The role of the behavioral sciences in environmental design, New York: Van Nostrand Reinhold, 1987.

[9] Mousavi, Mir Taher, Community involvement: a component of social capital, Journal of Social Welfare, No. 23, 2006, 67-92.

[10] Onyx, J., \& Bullen, P, Measuring social capital in five communities in NSW: An analysis. University of Technology, Sydney, Centre for Australian Community Organisations and Management (CACOM), 1997.

[11] Spellerberg, A, Framework for the measurement of social capital in New Zealand. Wellington: Statistics New Zealand, 2001.

[12] Tavassoli, Gh, The concept of capital in classical theories, social sciences letters, No. 26, 2005, 1-32.

[13] Mahdavinejad, M., Abedi, M. Community-Oriented Landscape Design for Sustainability in Architecture and Planning, Procedia Engineering, 2011, 21: 337-344.

[14] Mahdavinejad, M., Bemanian, M., Hajian, M., Pilechiha, P. Usage of Indigenous Architectural Patterns for Manufacturing Industrial Housing, Case: Renovation Project of Odlajan of Tehran, Iran, Advanced Materials Research, 2012, 548: 875-879.

[15] Mahdavinejad, M., Moradchelleh, A. Family-Led Method in Art Education and Learning, Case: Tehran, Iran, Middle-East Journal of Scientific Research, 2011, 9 (4): 554-560.

[16] Mahdavinejad, M. Rezaei Ashtiani, S., Ebrahimi, M., Shamshirband, M. Proposing a Flexible Approach to Architectural Design as a Tool for Achievement Eco-Friendly Multi-Purpose Buildings, Advanced Materials Research, 2012, 622-623: 1856 1859.

[17] Mahdavinejad, M., Mashayekhi, M., Ghaedi, A. Designing Communal Spaces in Residential Complexes, Procedia - Social and Behavioral Sciences, 2012, 51: 533-539.

[18] Chiesura A. The role of urban parks for sustainable city, Landscape and Urban Planning, 2004: 129-138.

[19] Sanoff H. Community participation methods in design and planning, John Wiley\& Sons, Inc.: 2000. 